\title{
Beweis des Satzes von Morley nach A. Connes
}

\author{
Hansjörg Geiges \\ Hansjörg Geiges wurde 1966 in Basel geboren und wuchs in der Faust-Stadt Staufen \\ im Breisgau auf. Ab 1985 studierte er Mathematik in Göttingen, Zürich, Bonn und \\ Cambridge, wo er 1992 promoviert wurde. Danach verbrachte er weitere akademi- \\ sche Wanderjahre in Stanford, Cambridge und an der ETH Zürich. Seit 1998 ist er \\ ordentlicher Professor an der Universität Leiden. Seine Forschungsinteressen liegen \\ in der Geometrie und Topologie.
}

\section{Der Satz von Morley}

Die meisten Sätze der euklidischen Geometrie, die im Unterricht der Sekundarstufe behandelt werden, finden sich bereits in den Elementen von Euklid [5]. Auch die Beweise, die im Unterricht vorgestellt werden, unterscheiden sich oft kaum von den klassischen Beweisen. Einer der bekanntesten „,neueren“ Sätze der Elementargeometrie ist der folgende von F. Morley um 1904 gefundene und bewiesene Satz.

Satz 1 Sei ABC ein Dreieck in der euklidischen Ebene. Dann formen die drei Schnittpunkte der benachbarten Winkeldreiteilenden der drei Winkel von ABC ein gleichseitiges Dreieck (siehe Abb. 1).

Überraschend ist schon die Tatsache, daß dieser hübsche Satz der Elementargeometrie erst so spät gefunden wurde. Wie kaum ein anderer Satz der euklidischen Geometrie hat er überdies professionelle Mathematiker und Amateure immer wieder dazu angeregt, neue Beweise (oder Verallgemeinerungen) zu suchen. Eine Reihe von Beweisen findet man zum Beispiel in [1] und [3], und eine umfassende Literaturliste in [6].

Man könnte glauben, daß Sätze der ebenen Geometrie nur dann nicht schon bei Euklid stehen, wenn sie nicht sonderlich elegant sind. Eine der wenigen (und bekanntesten) Ausnahmen ist der Satz von Morley, der besagt, daß die Schnittpunkte der benachbarten Winkeldreiteilenden eines beliebigen Dreiecks stets ein gleichseitiges Dreieck formen. Dieser überraschende Satz ist noch nicht einmal 100 Jahre alt. Nicht nur Hobby-Mathematiker haben sich seither immer wieder mit diesem Satz beschäftigt. Im vorliegenden Artikel wird eine elementare Darstellung eines auf der Geometrie der komplexen Zahlen beruhenden Beweises gegeben, der erst kürzlich von dem FieldsMedaillen-Gewinner Alain Connes gefunden wurde. 


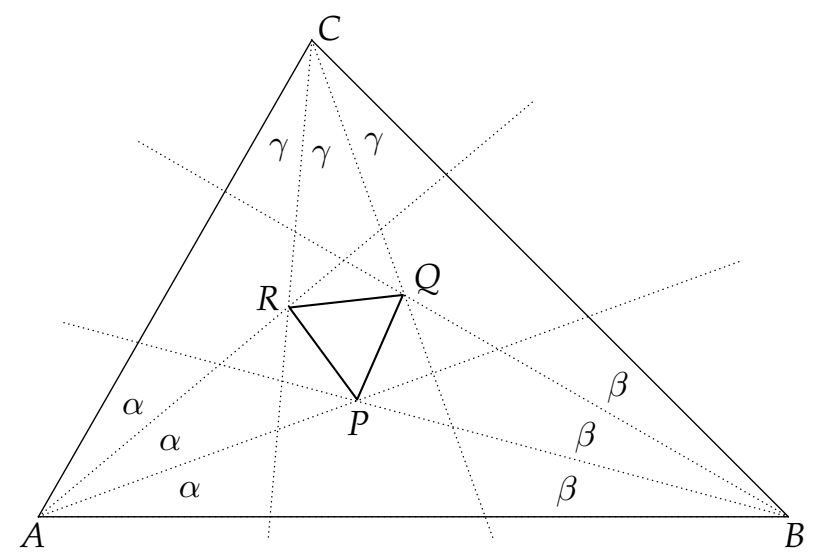

Abb. 1 Der Satz von Morley

Der neueste Beweis wurde vor wenigen Jahren von Alain Connes [2] gegeben. Connes ist einer der bekanntesten Mathematiker unserer Zeit. Er forscht am renommierten Institut des Hautes Études Scientifiques bei Paris, und im Jahre 1982 wurde er für sein Werk mit der Fields Medaille ausgezeichnet, dem „Nobelpreis für Mathematik“. Offensichtlich ist sich auch ein so bedeutender Mathematiker nicht zu schade dafür, hin und wieder einen frischen Blick auf Ergebnisse der Elementargeometrie zu werfen.

Der Beweis von Connes verwendet die geometrische Interpretation des Rechnens mit komplexen Zahlen, wobei Connes allerdings von einem höheren Standpunkt aus argumentiert. Mit dem vorliegenden Artikel möchte ich diesen Beweis in einer vereinfachten Version einem breiteren Publikum zugänglich machen. Mit geeigneter Vorbereitung läßt sich dieser selbst auf dem Niveau der Schulmathematik behandeln. Verglichen mit vielen anderen Darstellungen, die oft auf scheinbar blindem trigonometrischem Rechnen oder kniffligen geometrischen Konstruktionen beruhen, liegt der Vorteil des Beweises von Connes darin, daß die Beweisstrategie viel deutlicher zutage tritt.

\section{Komplexe Zahlen und ebene Geometrie}

Ich verwende im folgenden ohne weitere Erläuterung die komplexen Zahlen $\mathbb{C}$. Es sei aber darauf hingewiesen, daß sich die hier benötigten Eigenschaften auch elementar einführen lassen: Identifikation von $\mathbb{C}$ mit der euklidischen Ebene $\mathbb{R}^{2}$ als Punktmenge; Existenz von Addition und Multiplikation auf $\mathbb{C}$ und deren geometrische Interpretation in der euklidischen Ebene.

Für feste $a, b \in \mathbb{C}, a \neq 0$, ist die Abbildung $f(z)=a z$ eine Drehstreckung von $\mathbb{C}$ und $f(z)=z+b$ eine Translation. Eine allgemeine sogenannte affine Transformation von $\mathbb{C}$ ist von der Form $f(z)=a z+b$ mit $a \neq 0$; die Umkehrabbildung ist $f^{-1}(z)=a^{-1} z-a^{-1} b$. Für $a \neq 0,1$ hat $f$ den eindeutigen Fixpunkt

$$
F(f)=\frac{b}{1-a}
$$


Schreibt man $f$ (für $a \neq 0,1$ ) in der Form

$$
f(z)=a\left(z-\frac{b}{1-a}\right)+\frac{b}{1-a}
$$

so erkennt man $f$ als Drehstreckung mit Zentrum $F(f)$. Wir schreiben $\rho(f)=a$ für den Rotationsanteil von $f$ und $\tau(f)=b$ für den Translationsanteil. Für $f_{i}(z)=a_{i} z+b_{i}$, $i=1,2$, hat man dann

$$
\rho\left(f_{1} f_{2}\right)=a_{1} a_{2} \text { und } \tau\left(f_{1} f_{2}\right)=b_{1}+a_{1} b_{2},
$$

wobei die Komposition von Abbildungen wie üblich von rechts gelesen wird.

Anregungen zur ausführlicheren Behandlung dieser Punkte findet man in [4] und [7].

Lemma 2 Sei $\eta=(\cos (2 \pi / 3), \sin (2 \pi / 3)) \in \mathbb{R}^{2}=\mathbb{C}$. Dann gilt $1+\eta+\eta^{2}=0$.

Der Beweis folgt durch Inspektion von Abb. 2. Man beachte, daß auch $\eta^{2}$ dieser Gleichung genügt: $1+\eta^{2}+\left(\eta^{2}\right)^{2}=0$, denn $\eta^{3}=1$. Die komplexen Zahlen $\eta$ und $\eta^{2}$ sind die eindeutigen Lösungen von $z^{3}=1, z \neq 1$.

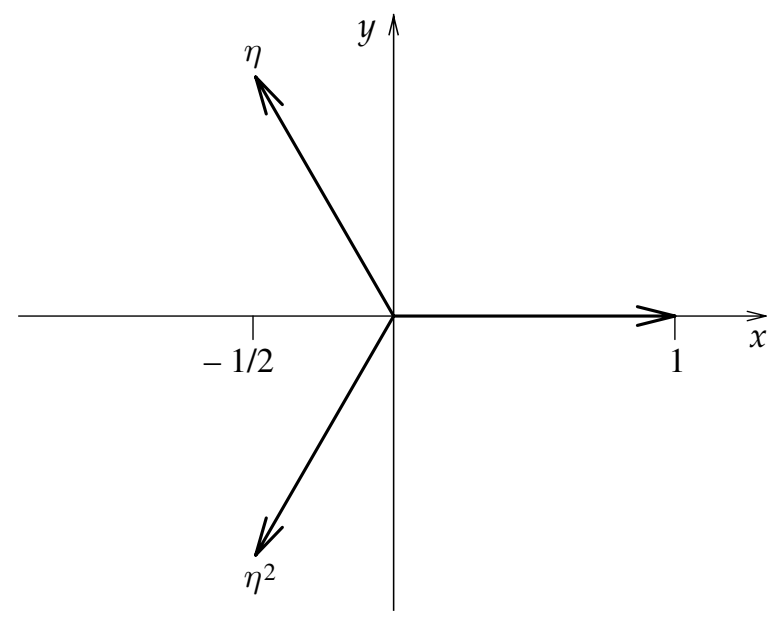

Abb. 2 Beweis von Lemma 2

Lemma 3 Ein Dreieck $P Q R$ in $\mathbb{R}^{2}=\mathbb{C}$ ist genau dann ein positiv (d.h. im Gegenuhrzeigersinn) orientiertes gleichseitiges Dreieck, wenn

$$
P+Q \eta+R \eta^{2}=0 \text {. }
$$

Beweis. Das Dreieck $P Q R$ ist gleichseitig genau dann, wenn das verschobene Dreieck $0(Q-P)(R-P)$ gleichseitig ist. Letzteres, zusammen mit der Orientierungsannahme, ist äquivalent $\mathrm{zu}$

$$
(Q-P)+(R-P) \eta=0,
$$

siehe Abb. 3. Mit Lemma 2 ist dies weiter äquivalent $\mathrm{zu}$

$$
P+Q \eta+R \eta^{2}=P\left(-\eta-\eta^{2}\right)+Q \eta+R \eta^{2}=((Q-P)+(R-P) \eta) \eta=0 .
$$




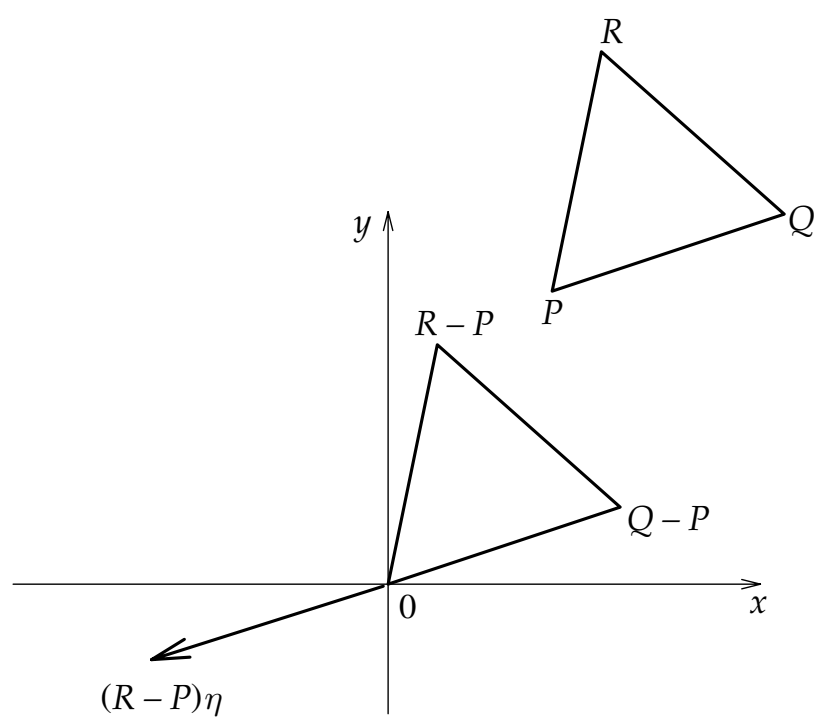

Abb. 3 Zum Beweis von Lemma 3

\section{Beweis des Satzes von Morley}

Es sei $f_{1}$ (bzw. $f_{2}, f_{3}$ ) die Drehung im Gegenuhrzeigersinn um den Punkt $A$ (bzw. $B, C$ ) um einen Winkel $2 \alpha$ (bzw. $2 \beta, 2 \gamma$ ), wobei die Winkel wie in Abb. 1 bezeichnet sind. Weiter bezeichnen wir für zwei Punkte $T \neq U$ in $\mathbb{C}$ mit $S_{T U}$ die Spiegelung an der durch $T$ und $U$ bestimmten Geraden. Dann gilt

$$
f_{1}^{3}=S_{A C} S_{A B}, \quad f_{2}^{3}=S_{A B} S_{B C}, \quad f_{3}^{3}=S_{B C} S_{A C} .
$$

Insbesondere folgt hieraus, da $\beta f_{1}^{3} f_{2}^{3} f_{3}^{3}$ die identische Abbildung ist. Man beachte weiter, da $\beta$ der Rotationsanteil von $f_{1} f_{2} f_{3}$ einer Rotation im Gegenuhrzeigersinn um $2 \alpha+2 \beta+$ $2 \gamma=2 \pi / 3$ entspricht, also $\rho\left(f_{1} f_{2} f_{3}\right)=\eta$.

Mit den Bezeichnungen aus Abschnitt 2 und $P, Q, R$ wie in Abb. 1 gilt

$$
P=F\left(f_{1} f_{2}\right), \quad Q=F\left(f_{2} f_{3}\right), \quad R=F\left(f_{3} f_{1}\right) .
$$

Es ist daher naheliegend zu fragen, ob die zu beweisende Beziehung zwischen $P, Q$ und $R$, nämlich die in Lemma 3, aus einer umfassenderen Aussage über Fixpunkte geeigneter affiner Abbildungen hergeleitet werden kann.

In der Tat folgt der Satz von Morley unmittelbar aus dem folgenden allgemeinen Satz über affine Transformationen von $\mathbb{C}$. Die dort gemachten Grundannahmen an die $f_{i}$ und Bedingung (i) sind nämlich im obigen speziellen Fall erfüllt.

Satz 4 Es seien

$$
\begin{aligned}
f_{i}: & \mathbb{C} \\
z & \longmapsto a_{i} z+b_{i}, a_{i} \neq 0,
\end{aligned}
$$


$i=1,2,3$, drei affine Transformationen von $\mathbb{C}$, die den Bedingungen

$$
a_{i} a_{j} \neq 1 \text { für } i \neq j \text { und } a_{1} a_{2} a_{3} \neq 1
$$

genügen, d.h. $f_{1} f_{2} f_{3}$ und die Komposition von je zwei der $f_{i}$ seien keine Translationen. Setze $\omega=\rho\left(f_{1} f_{2} f_{3}\right)=a_{1} a_{2} a_{3}$. Dann sind äquivalent:

(i) $f_{1}^{3} f_{2}^{3} f_{3}^{3}$ ist die identische Abbildung.

(ii) $\omega^{3}=1$ und $P+Q \omega+R \omega^{2}=0$, wo $P=F\left(f_{1} f_{2}\right), Q=F\left(f_{2} f_{3}\right)$ und $R=F\left(f_{3} f_{1}\right)$.

Beweis. Falls Bedingung (i) erfüllt ist, so gilt $\omega^{3}=\rho\left(f_{1}^{3} f_{2}^{3} f_{3}^{3}\right)=1$, also $\omega=\eta$ oder $\omega=\eta^{2}$. Unter dieser Annahme an $\omega$ rechnen wir wie folgt. Zunächst bemerken wir, daß

$$
P=\frac{\tau\left(f_{1} f_{2}\right)}{1-\rho\left(f_{1} f_{2}\right)}=\frac{a_{1} b_{2}+b_{1}}{1-a_{1} a_{2}}, \quad Q=\frac{a_{2} b_{3}+b_{2}}{1-a_{2} a_{3}}, \quad R=\frac{a_{3} b_{1}+b_{3}}{1-a_{3} a_{1}} .
$$

Da wir ein Kriterium dafür finden wollen, wann $P+Q \omega+R \omega^{2}$ verschwindet, bietet es sich an, diesen Ausdruck nennerfrei zu machen und zu vereinfachen. Der zusätzliche Faktor $-\omega^{2} a_{1}^{2} a_{2}$ in der folgenden Rechnung dient allein dazu, das Endergebnis in besonders einfacher Gestalt zu erhalten. Wir rechnen also

$$
\begin{aligned}
-\omega^{2} a_{1}^{2} a_{2}(1- & \left.a_{1} a_{2}\right)\left(1-a_{2} a_{3}\right)\left(1-a_{3} a_{1}\right)\left(P+Q \omega+R \omega^{2}\right)= \\
=- & \omega^{2} a_{1}^{2} a_{2}\left(\left(1-a_{2} a_{3}\right)\left(1-a_{3} a_{1}\right)\left(a_{1} b_{2}+b_{1}\right)\right. \\
& +\left(1-a_{3} a_{1}\right)\left(1-a_{1} a_{2}\right)\left(a_{2} b_{3}+b_{2}\right) a_{1} a_{2} a_{3} \\
& \left.+\left(1-a_{1} a_{2}\right)\left(1-a_{2} a_{3}\right)\left(a_{3} b_{1}+b_{3}\right) a_{1}^{2} a_{2}^{2} a_{3}^{2}\right) \\
=- & \omega^{2} a_{1}^{2} a_{2}\left(b _ { 1 } \left(1-a_{2} a_{3}-a_{1} a_{3}+a_{1} a_{2} a_{3}^{2}\right.\right. \\
& \left.+a_{1}^{2} a_{2}^{2} a_{3}^{3}-a_{1}^{3} a_{2}^{3} a_{3}^{3}-a_{1}^{2} a_{2}^{3} a_{3}^{4}+a_{1}^{3} a_{2}^{4} a_{3}^{4}\right) \\
+ & b_{2}\left(a_{1}-a_{1} a_{2} a_{3}-a_{1}^{2} a_{3}+a_{1}^{2} a_{2} a_{3}^{2}\right. \\
& \left.+a_{1} a_{2} a_{3}-a_{1}^{2} a_{2} a_{3}^{2}-a_{1}^{2} a_{2}^{2} a_{3}+a_{1}^{3} a_{2}^{2} a_{3}^{2}\right) \\
+ & b_{3}\left(a_{1} a_{2}^{2} a_{3}-a_{1}^{2} a_{2}^{2} a_{3}^{2}-a_{1}^{2} a_{2}^{3} a_{3}+a_{1}^{3} a_{2}^{3} a_{3}^{2}\right. \\
& \left.\left.+a_{1}^{2} a_{2}^{2} a_{3}^{2}-a_{1}^{3} a_{2}^{3} a_{3}^{2}-a_{1}^{2} a_{2}^{3} a_{3}^{3}+a_{1}^{3} a_{2}^{4} a_{3}^{3}\right)\right) \\
= & \omega^{2} a_{1}^{2} a_{2}\left(b_{1}\left(-a_{1} a_{3}+a_{1} a_{2} a_{3}^{2}+a_{1}^{2} a_{2}^{2} a_{3}^{3}-a_{1}^{2} a_{2}^{3} a_{3}^{4}\right)\right. \\
& +b_{2}\left(a_{1}-a_{1}^{2} a_{3}-a_{1}^{2} a_{2}^{2} a_{3}+a_{1}^{3} a_{2}^{2} a_{3}^{2}\right) \\
& \left.+b_{3}\left(a_{1} a_{2}^{2} a_{3}-a_{1}^{2} a_{2}^{3} a_{3}-a_{1}^{2} a_{2}^{3} a_{3}^{3}+a_{1}^{3} a_{2}^{4} a_{3}^{3}\right)\right) \\
= & b_{1}\left(a_{1}^{2}-\omega a_{1}-\omega^{2} a_{1}+1\right) \\
+ & b_{2} a_{1}^{3}\left(-\omega^{2} a_{2}+1+a_{2}^{2}-\omega a_{2}\right) \\
+ & b_{3}\left(a_{1} a_{2}\right)^{3}\left(-\omega^{2} a_{3}+1+a_{3}^{2}-\omega a_{3}\right) \\
= & b_{1}\left(1+a_{1}+a_{1}^{2}\right)+b_{2} a_{1}^{3}\left(1+a_{2}+a_{2}^{2}\right) \\
& \quad+b_{3}\left(a_{1} a_{2}\right)^{3}\left(1+a_{3}+a_{3}^{2}\right) \\
= & \left(f_{1}^{3} f_{2}^{3} f_{3}^{3}\right), \\
& \left.b_{2}, a_{1}\right)
\end{aligned}
$$

wobei für die vorletzte Gleichung Lemma 2 verwendet wurde. Dies beweist den Satz. 


\section{Literatur}

[1] M. Berger: Geometry I, Universitext, Springer-Verlag, Berlin 1987.

[2] A. Connes: A new proof of Morley's theorem, Les relations entre les mathématiques et la physique théorique, Inst. Hautes Études Sci., Bures-sur-Yvette (1998), 43-46.

[3] H.S.M. Coxeter und S.L. Greitzer: Zeitlose Geometrie, Klett Studienbücher Mathematik, Ernst-KlettVerlag, Stuttgart 1983.

[4] H.-D. Ebbinghaus et al.: Zahlen, Grundwissen Mathematik, Springer-Verlag, Berlin 1983.

[5] Euklid: Die Elemente (herausgegeben und übersetzt von C. Thaer), Wissenschaftliche Buchgesellschaft, Darmstadt 1980

[6] C. Lubin: A proof of Morley's theorem, Amer. Math. Monthly 62 (1955), 110-112.

[7] T. Needham: Visual Complex Analysis, Oxford University Press 1997.

\section{Hansjörg Geiges}

Mathematisch Instituut

Universiteit Leiden

Postbus 9512

NL-2300 RA Leiden, Niederlande

e-mail: geiges@math.leidenuniv.nl 